\title{
Global Agile Team Design: An Informing Science Perspective
}

\section{Jason H. Sharp \\ Tarleton State University, Stephenville, TX, USA}

\author{
Sherry D. Ryan and \\ Victor R. Prybutok \\ University of North Texas, \\ Denton, TX, USA
}

sherry.ryan@unt.edu

victor.prybutok@unt.edu

\begin{abstract}
As software development becomes increasingly globally distributed, a potential challenge for organizations using agile methodologies is the design of their development teams. Using a case study approach, Cohen's updated model of the Informing Science framework served as a means for framing the discussion of global agile team design related to the components of task, environmental context, and delivery channel. The results of the study provided a set of data-derived propositions that serve to inform organizations of practical design considerations and support the idea that global agile teams represent a complex relationship involving the informing environment, informing, and the informer.
\end{abstract}

Keywords: agile development methods, global software development, informing team environment, global agile team design

\section{Introduction}

Software development continues to be plagued with issues related to the completion of projects that fulfill user requirements on-time and within budget. Agile development, which is a set of software development methodologies based on the concepts of adaptability and iterative development, has been suggested as a way to alleviate these recurring problems (Abrahamson,

Warston, Sippon, \& Ronkainen, 2003). Agile development promotes collaboration between crossfunctional and self-organizing teams utilizing adaptive planning and development that is responsive to change.

Another concept that some organizations have embraced is developing software using global virtual teams. The benefits of drawing from

Material published as part of this publication, either on-line or in print, is copyrighted by the Informing Science Institute. Permission to make digital or paper copy of part or all of these works for personal or classroom use is granted without fee provided that the copies are not made or distributed for profit or commercial advantage AND that copies 1) bear this notice in full and 2) give the full citation on the first page. It is permissible to abstract these works so long as credit is given. To copy in all other cases or to republish or to post on a server or to redistribute to lists requires specific permission and payment of a fee. Contact Publisher@InformingScience.org to request redistribution permission. a large group of talented software developers available around the world, a 24-hour work schedule, and the ability to rapidly form and deploy virtual teams is a strong lure for organizations that want to address the challenges faced in software development (Battin, Crocker, Kreidler, \& Subramanian, 2001; Carmel \& Agarwal, 2001; Damian \& Moitra, 2006; Herbsleb \& Moitra, 2001). How- 
ever, there are many difficulties that need to be overcome to create a multicultural, multidisciplinary, informing environment in which teams can flourish (Battin et al., 2001).

Prior research has investigated how to combine these approaches (Agerfalk \& Fitzgerald, 2006; Holmstrom, Fitzgerald, Agerfalk, \& Conchuir, 2006; Jalali \& Wohlin, 2012; Ramesh, Cao, Mohan, \& Xu, 2006; Sarker, Munson, Sarker, \& Chakraborty, 2009; Sarker \& Sarker, 2009). An issue that arises is the actual design of teams utilizing this combined approach. While the software development process has been studied from an Informing Science perspective (Roussev \& Rousseva, 2004), this research specifically examines the design of global virtual teams employing agile software development methodologies via a case study through the lens of Informing Science (Cohen, 2009).

\section{Application of the Informing Science Framework}

Cohen (2009) presented an updated model of the Informing Science framework that draws upon Shannon and Weaver's (1949) communication model and Wilson's (1981) model as shown in Figure 1.

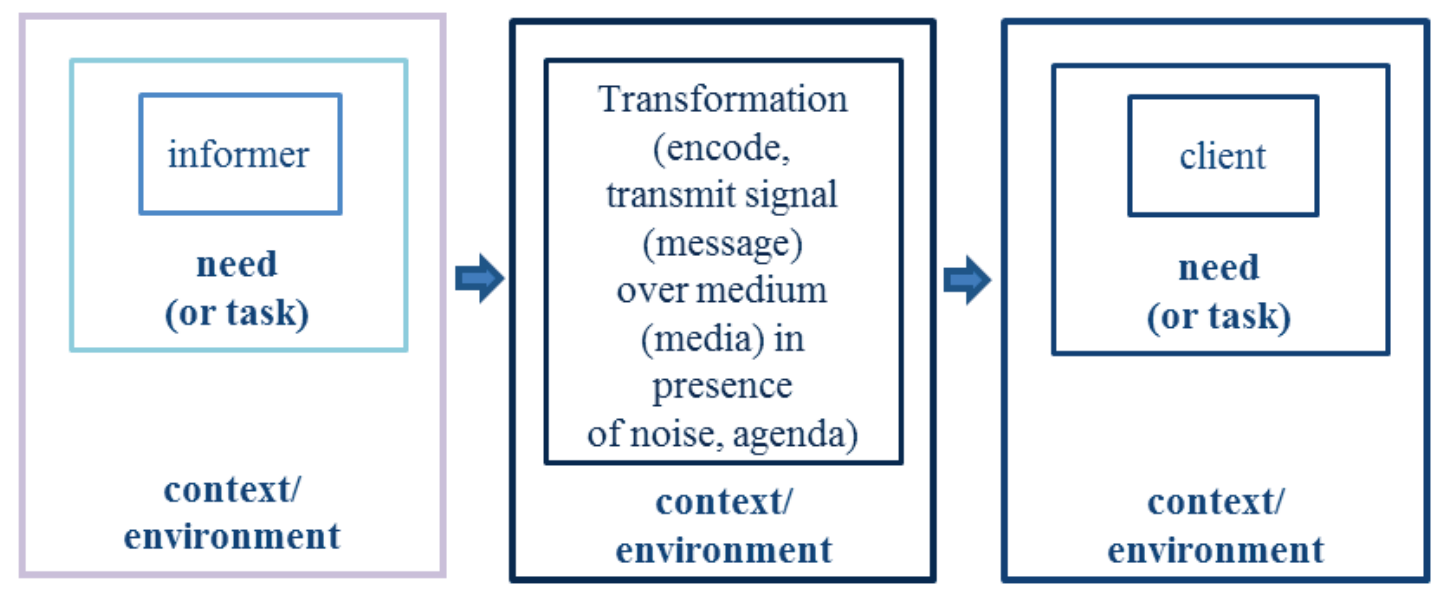

Figure 1: Cohen's (2009) Expanded Informing Science framework

In Figure 1, the informer is the sender (encoder) of a message, while the client is the receiver (decoder) of the message. In the global agile development arena, the informer and client can be members of the same global development team (either co-located or geographically dispersed) or the customer for whom the system is being developed.

Next in the figure is the need or task. In this research, the task was the software development that must be accomplished. Agile methods adhere in varying degrees to the values, principles, and practices set forth in the Agile Manifesto. The values emphasize "individuals and interactions over processes and tools," "working software over comprehensive documentation," "customer collaboration over contract negotiation," and "responding to change over following a plan" (Fowler \& Highsmith, 2001). Table 1 lists the twelve principles behind the Agile Manifesto. 


\section{Table 1: Twelve Principles Behind the Agile Manifesto}

\section{Principles}

1. Our highest priority is to satisfy the customer through early and continuous delivery of valuable software.

2. Business people and developers must work together daily throughout the project.

3. The most efficient and effective method of conveying information to and within a development team is face-to-face conversation.

4. Build projects around motivated individuals. Give them the environment and support they need, and trust them to get the job done.

5. The best architectures, requirements, and designs emerge from self-organizing teams.

6. At regular intervals, the team reflects on how to become more effective, then tunes and adjusts its behavior accordingly.

7. Deliver working software frequently, from a couple of weeks to a couple of months, with a preference to the shorter timescale.

8. Simplicity - the art of maximizing the amount of work not done — is essential.

9. Welcome changing requirements, even late in development. Agile processes harness change for the customer's competitive advantage.

10. Working software is the primary measure of progress.

11. Continuous attention to technical excellence and good design enhances agility.

12. Agile processes promote sustainable development. The sponsors, developers, and users should be able to maintain a constant pace indefinitely.

Specific agile practices depend on the agile methodology employed. For example, eXtreme Programming, one used agile methodology frequently, emphasizes small releases (a release every two weeks), pair programming (programmers working in pairs at the same computer where one person controls the keyboard and mouse while the other observes), continuous integration (checking in code and integrating multiple times per day), and user experience (documenting needs by writing a few words on an index card as a reminder of the discussion with the user) just to name a few (Beck \& Andres, 2005; Martin, 2003). Another example of an agile methodology that emphasizes specific agile practices is Scrum. These practices include sprints (an iteration or release of software approximately once a calendar month), the daily scrum (a meeting held each day of the sprint lasting 15 minutes or less), sprint planning (used to determine which product backlog subsets are most important and should be included in the next sprint), and scrum of scrums (the coordination of multiple Scrum teams) (Rubin, 2013; Schwaber \& Beedle, 2002).

Third, in Figure 1, is the context/environment. The initial Informing Science model was expanded to acknowledge explicitly that both the informer and the client exist within complex environments. In a global team environment, this environmental context can be conceptualized at two levels: the team context and the virtual context. When looking at global agile team configuration, previous literature has proposed that within a virtual context, boundary spanning and temporal distribution may impact processes, core norms, composition, and task design (Sharp \& Ryan, 2011). 
Transformation (the second box shown in Figure 1) relates to the delivery channel through which information is communicated and is essential to creating a productive informing environment. This typically refers to information and communication technologies (ICT), but transformation can also refer to specific interpersonal communication mechanisms. Although prior research explored the alignment between ICT users and managers from an Informing Science perspective (Fardal, 2007), this study focuses on the use of ICT between members of a global agile software development team. Because most members are not co-located, ICT is critical to project success.

This research is about designing successful global agile teams. While the psychological characteristics and fragilities of individual informers and clients are important, the scope of this particular study is limited to the task, environmental context, and delivery channel components.

\section{Research Method}

\section{Identification and Selection of Organizations and Teams}

A case study involving three U.S. based organizations was conducted. These organizations were identified based upon the following criteria: (1) the organization had globally distributed sites; (2) the organization was using an agile methodology to some degree; and (3) the organization was willing to participate in the study. Two of the organizations were multi-national, while the third used offshore contractors as a part of its team structure. In an effort to maximize the number and diversity of team designs, multiple organizations were included. While the organizations were similar in terms of industry, global distribution, and use of an agile methodology, there were differences among the teams themselves in regards to size, structure, and degree to which the agile methodology was implemented. A brief description of each organization is provided below:

Organization A consisted of about 135,000 employees across the globe. In addition to its own employees, it also hired contractors from several other countries. In terms of this organization's software development, a structured approach was used predominately, although small numbers of both individuals and teams were slowly adopting agile values, principles, and practices.

Organization B employed about 40,000 employees, distributing work between its onshore project management team and its offshore development team. While North America had been its primary focus, opportunities for expansion into the global environment were growing. This organization implemented a traditionally structured approach to software development for its internal customer application. However, Organization B began offering informal training to explore the values, principles, and practices of the agile approach, which generated interest among its information technology division.

Finally, Organization C employed around 9,000 employees located in 45 countries. Unlike Organizations $\mathrm{A}$ and $\mathrm{B}$, Organization $\mathrm{C}$ implemented organization-wide agile development which was highly supported by the top-level leadership. In fact, all employees were required to complete mandatory training in agile development.

After identifying the organizations outlined above, the identification and selection of teams to serve as cases was conducted. To select the individual cases, purposeful and snowball sampling techniques were used (Patton, 2002). Criteria for the sampling were as follows: (1) the team was formally or informally using an agile methodology; and (2) team members were globally distributed. A total of five teams were selected from across the three organizations located in various countries, including Australia, Brazil, India, Mexico, Poland, and the United States. In all 37 teams, members serving in various roles (developers, project managers, architects, technical leads, business analysts, quality assurance analysts, and test analysts) were included in the study. 


\section{Data Collection and Analysis}

Data were collected via semi-structured telephone interviews ranging from 25 minutes to 1 hour and 45 minutes. While all team members were potentially rich sources of data, several challenges (including scheduling and fluency in English) prevented the interviewing of every member from each team. Therefore, project managers from each team provided guidance with identifying relevant team members. This input generated a diverse cross-section of team members in terms of roles and responsibilities on the team. After prospective interview candidates were identified, each candidate received an email that explained the study and asked for voluntary participation.

Interview and case study protocols were developed prior to the interviews, and both served as guides throughout each phone interview (Yin, 2003). The interview protocol consisted of two sets of questions: one set for the project manager and one set for team members. The interview protocol guided the interview process after participants were asked a series of demographic questions. The case study protocol ensured consistency among all interviews, thus increasing the reliability of data collected during this case study.

To analyze data, case descriptions were developed to organize each case study. As is common in case study research, within-case and cross-case analyses were utilized. Within-case analyses were conducted first in order to become familiar with each case individually. Cross-case analyses were conducted next and were intended to go deeper into the cases' emerging themes and identify patterns shared by each individual case (Miles \& Huberman, 1984; Yin, 2003). All data were analyzed using MAXQDA, a qualitative data analysis software package. Using these data analysis techniques, nine propositions were developed and are described in the following section.

\section{Proposition Development}

From the within-case and cross-case analyses, "overall impressions, tentative themes, concepts, and possibly even relationships between variables begin to emerge" (Eisenhardt, 1989, p. 541). To determine how to design successful agile teams in globally distributed environments, the Informing Science framework components of task, environmental context, and delivery channel were considered, and data-derived propositions were developed for each. According to Pare (2002), propositions may define an initial set of laws characterizing the interaction and relationships. The objective, therefore, is to identify and generalize issues of the Informing Science framework components mentioned above that occurred in successful global agile team designs. The results are presented below by component.

\section{Task}

Agile practices, such as short iterations and small releases, helped foster an overall sense of meaningfulness as members are able to focus both on the smaller components of the project as well as envision the overall goal. As one member commented:

I think it is important for everybody on the team to know the full scope of the project as well ... because I think it only helps the team success if everybody is aware of what the project entails... not just maybe the area that they are involved in, but really what is going on in the whole project.

Another member stated:

Shorter iterations are the important factor because successfully completing those small iterations leads to overall project success.

Designing tasks with short iterations and small releases may decrease complexity, provide small victories for the team, help members to keep the 'big picture' in sight by letting them see the 
smaller parts being completed, and help motivation by keeping members from being overwhelmed by the size and complexity of the project.

The distribution of work among the team was also a consideration. The data indicated that two teams in two different locations should not be working on the same component or feature. As one member noted:

I think it makes a lot more sense to have a single team that's geographically together having them working on that component, that feature, so that they are having that discussion among themselves in terms of what does this mean, what's the right way to do this, how do we test it, that's all pretty much local there instead of having to have those conversations among multiple locations.

Thus, the following is proposed:

Proposition 1: A successful globally distributed agile team configuration uses agile practices, such as short iterations and small releases, to facilitate meaningfulness and motivation through achieving small victories and alleviating the "overwhelmingness" that may accompany complex projects.

An analysis of the responses from each team in regard to the extent of agility for adhering to the values of the Agile Manifesto suggested that these values can be implemented successfully in a globally distributed environment. In this same manner, the analysis of the responses from each team in regard to the extent of agility for adhering to the principles of the Agile Manifesto indicated that with the obvious exception of Principle 6, it was possible to implement successfully the other principles in a globally distributed environment.

Proposition 2: A successful globally distributed agile team configuration adheres to the "spirit" of the values and principles of the Agile Manifesto either directly or indirectly, although individual members may not be familiar with the Agile Manifesto in its official form.

\section{Context/Environment}

\section{Team structure}

A smaller team was identified as more beneficial for a successful configuration, as the size of the team had an impact on the implementation of certain agile practices. As one member stated, "smaller teams are always preferable to larger teams." Another member suggested:

The ideal team size is something less than twenty . . . if you have a very large team trying to build a single piece of software it becomes an enormous challenge to keep everybody on the same page.

Practices such as the daily stand-up meeting (a brief meeting designed to provide a status update to the team members) and iteration planning (Martin, 2003) began to lose their focus as the size of the team grew. Overall, there was less participation and interest during the meetings.

If the teams became too large, teams divided into sub-teams and held multiple stand-up meetings and iteration planning sessions. Additionally, as the teams grew larger, members collected more user stories. During meetings, team members spent more time sharing these user stories, which decreased the agility of the team. One member summed it up by saying, "if it is a smaller team it is a little bit easier to control and, you know, manage than a larger team." Thus, the following is proposed:

Proposition 3: A successful globally distributed agile team configuration consists of a smaller number of members to facilitate better certain agile practices, such as the daily 
stand-up meeting, iteration planning, iteration demos, iteration retrospectives, and user stories.

Data also suggested that the use of an agile methodology can ease new members' transition to the team. Teamwork, collaboration, and strong technical skills are hallmarks of agile methodologies, so it was important to call upon the expertise of existing members to assist new or less experienced members. One member noted the importance of task and knowledge-related skills by stating:

For sure they are important, however, I would say that something we discovered some time ago in this agile approach that agile brought very new team members to be fully efficient in the team in a very short period.

For example, the use of pair programming allowed a less-experienced member to team up with a more seasoned member and work together until the less-experienced member was comfortable with how things within the team worked. Thus, the following is proposed:

Proposition 4: A successful globally distributed agile team configuration takes advantage of agile practices that promote teamwork and help new or less experienced members to increase their knowledge and task-related skills.

Another team structure-related issue involved how human resources were allocated to the team on a monthly basis. Due to contractual issues, resources were often allocated month-to-month, including the determination of the number of people working for a client in the months to come. In some cases, the contract required a minimum number of people to work on a particular area for a particular client. This presented a particular challenge when contractual mandates were combined with a six-week iteration period. As one member commented:

... we don't really have any process in place to formally sync that up with our monthly-man loading requirements and it's difficult ... you don't necessarily know . . you cannot guarantee that you will have the same people next month that you have this month, you just cannot guarantee that.

It was suggested, therefore, that the iteration cycle and monthly man-loading cycle be synchronized to minimize members from rotating off the team in the middle of an iteration. Thus, the following is proposed:

Proposition 5: A successful globally distributed agile team configuration synchronizes the iteration cycle and the monthly man-loading cycle so that members are not rotating off the team in the middle of an iteration.

\section{Virtual context issues}

Overall, the teams cited differences in holidays, language issues (such as accents, word choice and meaning, interpretation), and views about overtime and sick leave as cultural boundaries. The overall consensus was that crossing these cultural boundaries was not a significant deterrent to a successful team configuration. As one member indicated, the "world is getting smaller." Thus, in terms of boundary spanning, the following is proposed:

Proposition 6: A successful globally distributed agile team configuration may cross multiple functional, organizational, and cultural boundaries without negative affect, but organizational "buy-in" and the use of an agile process by other functional units are beneficial.

Data also suggested that geographic distance was not nearly as troublesome as time zone differences. Although there were benefits, such as continuous coverage of the project by handing off 
work, the overall feeling was that having some degree of overlap in work hours between sites was important to a successful configuration. As one team member commented:

I would say the biggest one was really just setting up meeting times when you have so many time zones ... sometimes it is four hours different, and the sometimes three hours, and sometimes two hours and to me was just time zones of trying to get . . find a time where everybody was around.

Limited overlap in work hours hindered access to members, decreased timely communication, lessened a sense of "teamness," and caused difficulty with the scheduling of meetings. All of these challenges presented a problem in terms of agile design since it emphasizes on-going collaboration, regular communication, and daily stand-up meetings. Thus, the following is proposed:

Proposition 7: A successful globally distributed agile team configuration provides at least some degree of overlapping work hours between sites to facilitate the use of agile values, principles, and practices.

\section{Delivery Channel}

All of the teams participating in this study utilized some measure of ICT. Overall, it was agreed that teleconferencing, instant messaging, desktop sharing, and collaboration software were essential to team configuration in relation to temporal distribution. Specifically, teleconferencing and instant messaging were primary means of conducting daily-standup meetings, iteration planning, iteration demos, and iteration retrospectives. As one member indicated, "we wouldn't be able to function with conference calls." Another member cited the importance of instant messaging "because it is hard to sometime pick up the phone and call someone from another country." Finally, another member stated, "definitely, the instant message is what we are using most often because it is cheaper than telephone and is instant, okay."

Desktop sharing was used to facilitate iteration demos, iteration planning, code review, and idea exchange. The teams reported limited use of videoconferencing due to difficulty in ease of use, complicated setup, and inadequate band-width. Thus, the following is proposed:

Proposition 8: A successful globally distributed agile team configuration makes use of multiple communication channels through the use of teleconferencing, instant messaging, desktop sharing, groupware, and email technologies.

The use of agile design increases the importance of interpersonal skills due to the fact that agile design relies heavily on daily interaction between members. Agile design is predicated on the concepts of communication, interactions, people, and collaboration. Thus, it was noted that strong interpersonal skills contributed to agile practices such as the daily stand-up meeting, which was conducted via ICT rather than face-to-face. One team member commented:

Oh, it is huge. Yeah. And I think, again, because it's . . the agile process is such a collaborative effort that, that builds those relationships, those interpersonal skills because you are not talking to somebody every two weeks, you are talking to somebody every day.

Because agile practices involved daily interaction, it was suggested that an agile methodology can actually help foster stronger interpersonal relationships. Thus, the following is proposed:

Proposition 9: A successful globally distributed agile team configuration takes advantage of the agile practices that emphasize daily interaction and utilize these practices to foster stronger interpersonal relationships. 


\section{Discussion}

Cohen (2009) stated that the "information needs of an individual are complex and are a function of context, environment, social or job role or task, and the individual's psychology" (p. 8). This statement is relevant when designing global agile teams. The propositions developed above sought to address agile design considerations from the perspective of the Informing Science framework specifically related to task, environmental context, and delivery channel components.

\section{Task}

Data revealed that some agile practices, such as short iterations and small releases, helped to alleviate the complexity of the task design. These practices are important especially within globally distributed environments where facilitating effective communication and coordination is complicated often by geographic distance. The effective transmission of information between the informer and client is vital. Within the overall task of software development, data showed that the implementation of agile practices (either indirectly or directly) was possible within a global environment. This could take the form of adopting a formal agile method in its entirety, tailoring select agile practices, or simply adhering to the "spirit" of agile design. Considering that a key component of agile design is communication, the implementation of an agile method to any degree has a potential benefit.

\section{Environmental Context}

Data showed that issues related to team structure benefited the design of the team. Key design elements (for instance, keeping the team small) helped facilitate specific agile practices, such as the daily standup meeting, iteration planning, iteration demos, iteration retrospectives, and user stories. Collaboration (such as sharing information between team members) was improved through the use of pair-programming, as new members were able to work with seasoned members to reduce project learning curves and increase their knowledge and task-related skills. The synchronization of the iteration cycle was found to be an important design element in regard to the virtual context. Ensuring that members did not rotate off the team in the middle of an iteration, thus leaving a knowledge gap to be filled by the incoming member, was crucial. Data showed that functional, organizational, and cultural boundaries did not need to hamper the design of a global agile team. Data indicated also that having the support of the organization and other functional units employing agile design was an advantage. Considering that agile design promotes ongoing collaboration and regular communication through practices such as daily stand-up meetings, iteration planning, 'demos', and iteration retrospectives, this study revealed that some degree of overlapping work hours across distributed sites was very important. If there is no time for members to communicate synchronously, then the transmission of information between informer and client is hampered.

\section{Delivery Channel}

The use of multiple ICTs as a delivery channel was essential. Teleconferencing and instant messaging were found to be the primary means for facilitating numerous agile practices. Related to the environmental context, it was important for the team to have overlapping time to communicate via ICT to improve communication and collaboration. Another finding to improve the transmission of information between informer and client was the use of certain agile practices to promote daily interaction and improve interpersonal relationships. 


\section{Limitations and Future Research}

While the propositions above were developed from an analysis of data collected during the interview process and provide insights into the design of global agile teams through the Informing Science framework, the authors recognize that certain limitations do exist. First, the cases examined were perceived as successful designs by the team members. However, there is a possibility that data collected based upon participants' perceptions were skewed and/or biased and did not represent actual reality. This is a common limitation when the data is self-reported, and this should be taken into consideration. Because the interviews were recorded digitally, participants may not have been as forthcoming with data that they reported. Second, while every effort was made to define terms, some of the terminology used in the interview protocol may not have been as familiar to some participants as to others due to cultural and language differences. Third, as Yin (2003) suggested, the data analysis procedures associated with the case study method relied "strongly on argumentative interpretation, not numeric tallies." Therefore, the challenge for the researcher was to "know how to develop strong, plausible, and fair arguments that are supported by the data" (p. 135). However, adherence to a predesigned analysis protocol as described earlier helps to minimize this concern. Finally, a traditional limitation cited for case study research relates to the generalizability of the findings. As such, this study does not provide statistical generalization where "an inference is made about a population (or universe) on the basis of empirical data collected about a sample" (Yin, 2003, p. 32); rather, the goal of case study research is analytical generalization, which involves generalization to "theoretical propositions and not to populations or universes" (p. 10).

The scope of this research focused on the Informing Science framework elements of task, environmental context, and delivery channel. However, future research on global agile team design should attempt to address "the less studied areas of Informing Science," such as the psychological, physiological, behavioral, and social aspects related to team design. It could also address what Cohen (2009) defined as "fragility": the "cognitive limitations of human processing of information" (p. 8). Global agile teams are made up of individuals, and when only task, environment context, and delivery channel are considered, an extremely important element is missing: people. For example, what are the human-related issues that influence the transmission of information between the informer and client within a global agile team? Is a certain personality type better suited to work within this type of team? What should the team composition be comprised of in terms of knowledge and skills? Are there specific norms expected in these types of teams? How long had the team been in existence? How had their expectations and procedures changed? What was the motivating factor for becoming a global agile team?

This research consisted of qualitative interviews, which fostered depth of insight into a phenomenon. Based upon analyses of the interviews, propositions were developed to enlarge the understanding of how global agile teams can best be configured. Using this research as a starting point, further research should confirm these propositions through quantitative empirical research, such as a large scale survey.

\section{Conclusion}

As the proposition development above indicates, the Informing Science framework components of task, environmental context, and delivery channel are beneficial for framing the discussion of global agile team design. These components provide organizations with a foundation for considering the implementation of agile methodologies within their existing global virtual teams or the design of new teams. This research provided the first study to apply the Informing Science framework to the area of global agile team design. Due to the complexity of designing global teams, it is crucial to call upon multiple fields to inform successful team design, especially when 
the team employs an agile methodology. Global agile team design relies on a variety of literature in the areas of work group design, virtual teams, global software development, and agile development. As noted by Cohen (2009), this "gets to the heart" of the Informing Science philosophy, which is the "transfer of knowledge from one field to another: breaking down disciplinary boundaries that hinder the flow of knowledge" (p. 1).

Findings from this study supported the contention that there are a complex set of relationships that involve the informing environment, informing, and the informer. For example, the mechanics of establishing the informing environment within the agile team environment, such as keeping the team small to facilitate agile practices, also sets the tone for the environment. In addition, pairing new team members with seasoned members to enhance the learning curve of new members also defines the environment. The importance of these practices, along with technology's impact on the success of agile teams, proves the need for further research into these relationships and interactions. We believe that the findings of this study suggest that agile teams provide a unique opportunity to conduct such research.

\section{References}

Abrahamson, P., Warsta, J., Sippon, S. T., \& Ronkainen, J. (2003). New directions on agile methods: A comparative analysis. Proceedings of the $25^{\text {th }}$ International Conference on Software Engineering, 244254.

Agerfalk, P. J., \& Fitzgerald, B. (2006). Flexible and distributed software processes: Old petunias in new bowls? Communications of the ACM, 49(10), 27-34.

Battin, R. D., Crocker, R., Kreidler, J., \& Subramanian, K. (2001). Leveraging resources in global software development. IEEE Software, 18(2), 70-77.

Beck, K., \& Andres, C. (2005). Extreme programming explained: Embrace change (2 ${ }^{\text {nd }}$ ed.). Boston, MA: Addison-Wesley.

Cohen, E. B. (2009). A philosophy of informing science. Informing Science: The International Journal of an Emerging Transdiscipline, 12, 1-15. Retrieved from http://www.inform.nu/Articles/Vol12/ISJv12p001-015Cohen399.pdf

Carmel, E., \& Agarwal, R. (2001). Tactical approaches for alleviating distance in global software development. IEEE Software, 18(2), 22-29.

Damian, D., \& Moitra, D. (2006). Global software development: How far have we come? IEEE Software, 23(5), 17-19.

Eisenhardt, K. M. (1989). Building theories from case study research. The Academy of Management Review, 14(4), 532-550.

Fardal, H. (2007). ICT strategy in an ICT user perspective: Exploring alignment between ICT users and managers. Issues in Informing Science and Information Technology, 4, 661-673.

Fowler, M., \& Highsmith, J. (2001). The agile manifesto. Retrieved February 17, 2014, from http://www.agilemanifesto.org

Herbsleb, J. D., \& Moitra, D. (2001). Global software development. IEEE Software, 18(2), 16-20.

Holmstrom, H., Fitzgerald, B., Agerfalk, P. J., \& Conchuir, E. O. (2006). Agile practices reduce distance in global software development. Information Systems Management, 23(3), 7-18.

Jalali, S., \& Wohlin, C. (2012). Global software engineering and agile practices: A systematic review. Journal of Software: Evolution \& Process, 24(6), 643-659.

Martin, R. C. (2003). Agile software development: Principles, patterns, and practices. Upper Saddle River, NJ: Prentice-Hall.

Miles, M. B., \& Huberman, A. M. (1984). Qualitative data analysis. Beverly Hills, CA: Sage Publications. 
Pare, G. (2002). Enhancing rigor of qualitative research: Application of a case methodology to build theories of IT implementation. The Qualitative Report, 7(4).

Patton, M. Q. (2002). Qualitative Research and Evaluation methods (3rd ed.). Thousand Oaks, CA: Sage Publications.

Ramesh, B., Cao, L., Mohan, K., \& Xu, P. (2006). Can distributed software development be agile? Communications of the ACM, 49(10), 41-46.

Roussev, B., \& Rousseva, Y. (2004). Software development: Informing sciences perspective. Issues in Informing Science and Information Technology, 1, 237-245.

Rubin, K. S. (2013). Essential Scrum: A practical guide to the most popular agile process. Upper Saddle River, NJ: Addison-Wesley.

Sarker, S., Munson, C. L., Sarker, S., \& Chakraborty, S. (2009). Assessing the relative contribution of the facets of agility to distributed systems development success: An Analytic Hierarchy Process approach. Journal of Information Systems, 18, 285-299.

Sarker, S., \& Sarker, S. (2009). Exploring agility in distributed information systems development teams: An interpretive study in an offshoring context. Information Systems Research, 20(3), 440-461.

Schwaber, K., \& Beedle, M. (2002). Agile software development with scrum. Upper Saddle River, NJ: Prentice-Hall.

Shannon, C. E., \& Weaver, W. (1949). The mathematical theory of communication. Urbana, Illinois: The University of Illinois Press.

Sharp, J. H., \& Ryan, S. D. (2011). Global agile team configuration. Journal of Strategic Innovation and Sustainability, 7(1), 120-134.

Wilson, T. D. (1981). On user studies and information needs. Journal of Documentation, 37(1), 3-15.

Yin, R. K. (2003). Case study research: Design and methods (3rd ed.). Thousand Oaks: Sage Publications.

\section{Biographies}

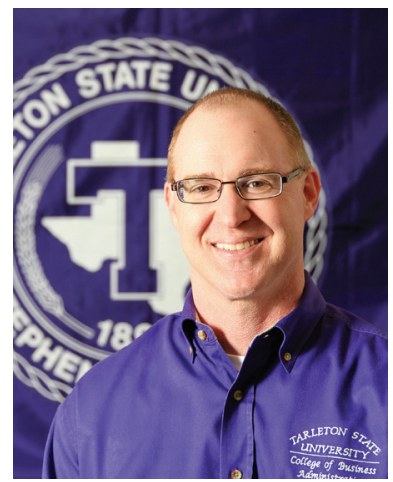

Jason H. Sharp is an Associate Professor of Computer Information Systems at Tarleton State University. He received his Ph.D. from the University of North Texas. Prior to entering academia, he worked as a systems support specialist and developed custom database solutions. His research interests include agile development, social media, and instructional technology. His work has appeared in such journals as: Issues in Informing Science and Information Technology, Journal of Information Technology Education: Innovations in Practice, The DATA BASE for Advances in Information Systems, and Journal of Information Technology Management. Jason's email is jsharp@tarleton.edu 


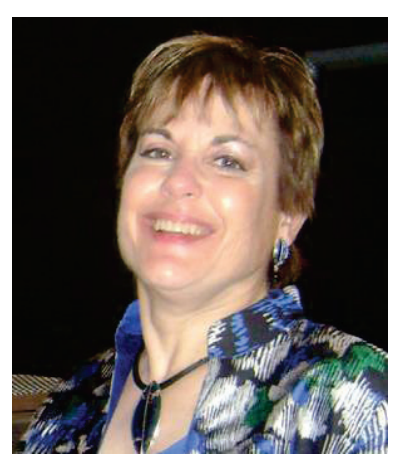

Sherry D. Ryan is an Associate Professor of Information Technology and Decision Sciences at the University of North Texas. She received her Ph.D. in IS from the University of Texas at Arlington and an MBA from the University of Southern California. Prior to returning to academia she worked for IBM, teaching courses and speaking at national conferences. Her research interests include IT human resource issues, knowledge management, and social media. Her work has appeared in journals including Journal of Management Information Systems, Decision Support Systems, and Informing Science: the International Journal of an Emerging Transdiscipline. Sherry's email is sherry.ryan@unt.edu

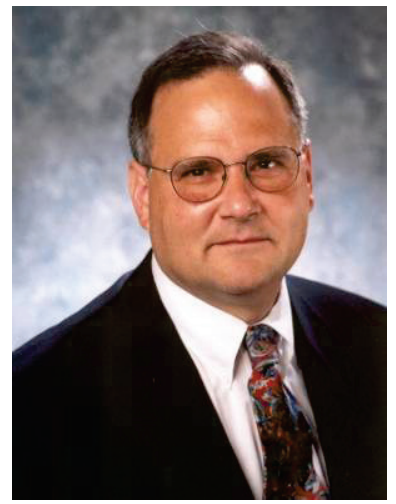

Victor R. Prybutok is a Regents Professor of Decision Sciences in the Information Technology and Decision Sciences Department and Associate Dean of the Toulouse Graduate School at the University of North Texas. He received, from Drexel University, his B.S. with High Honors in 1974, a M.S. in Bio-Mathematics in 1976, a M.S. in Environmental Health in 1980, and a Ph.D. in Environmental Analysis and Applied Statistics in 1984. Dr. Prybutok is an American Society of Quality certified quality engineer, certified quality auditor, certified manager of quality / organizational excellence, and an accredited professional statistician (PSTAT ${ }^{\circledR}$ ) by the American Statistical Association. Dr. Prybutok has authored over 150 journal articles, several book chapters, and more than 150 conference presentations in information systems measurement, quality control, risk assessment, and applied statistics. In addition, he serves on the editorial board of the Quality Management Journal. Victor's email is victor.prybutok@unt.edu 